\title{
CONSTRUCCIÓN Y AJUSTE GEOGRÁFICO DE DATOS ESPACIALES DE SISTEMAS DE AGUA POTABLE Y SANEAMIENTO DE AYA EN COSTA RICA
}

\author{
CONSTRUCTION AND GEOGRAPHIC SETTING OF \\ SPATIAL DATA OF DRINKING WATER SUPPLY AND \\ SANITATION -AYA, IN COSTA RICA
}

\author{
Esteban Alberto González-Ramírez ${ }^{1}$ \\ Instituto Costarricense de Acueductos y Alcantarillados, Costa Rica
}

\begin{abstract}
RESUMEN
En el documento, se presenta el proceso y los resultados del proyecto "construcción y ajuste geográfico de datos espaciales de los sistemas de agua potable y saneamiento del Instituto Costarricense de Acueductos y Alcantarillados", realizado mediante el uso de Sistemas de Información Geográfica. Se detalla la manera en que se realizó la organización de información territorial de infraestructuras de acueductos y alcantarillados, los métodos usados en la construcción y ajuste espacial de dichos datos, pertenecientes a ciudades intermedias y rurales (periféricas) de Costa Rica, los resultados obtenidos del proyecto y su aplicación en diversos campos técnicos y operativos.
\end{abstract}

Palabras clave: acueductos, sistemas de saneamiento, infraestructuras, Sistemas de Información Geográfica, dato.

\section{ABSTRACT}

The document presents the process and results of the project "Construction and Geographical Setting of Spatial Data of Drinking Water and Sanitation Systems of the Costa Rican Institute of Aqueducts and Sewers" performed by using Geographic Information Systems. It also presents details

1 Licenciado en Ciencias Geográficas, pertenece a la Subgerencia de Ambiente, Investigación y Desarrollo del Instituto Costarricense de Acueductos y Alcantarillados, San José, Costa Rica. Correo electrónico: egonzalez@aya.go.cr

Fecha de recepción:4 de noviembre de 2014

Fecha de aceptación: 3 de marzo de 2015 
Esteban Alberto González-Ramírez. Construction and geographic setting of spatial

data of drinking water supply and sanitation-AyA, in Costa Rica

on how to organize territorial information regarding water and sewer infrastructure, as well as the methods used in the construction and spatial setting of the data that belong to intermediate and rural (peripheral) cities in Costa Rica. Furthermore, it presents the results obtained from the project, and their use in different technical and operational fields.

Keywords: aqueducts, sanitation systems, infrastructure, Geographic Information Systems, fact.

\section{Introducción}

La información territorial resulta esencial en la toma de decisiones por parte de las organizaciones, ya que permite integrar y coordinar diversos procesos de un determinado negocio. En el caso del Instituto Costarricense de Acueductos y Alcantarillados (AyA), la ubicación territorial de la infraestructura civil, que permite el trasiego de agua para el abastecimiento de áreas urbanas y de saneamiento y su representación gráfica mediante mapas, resulta fundamental para cumplir parte de los objetivos por los que fue creada la institución.

En las últimas décadas, en el AyA se ha implementado el uso de herramientas SIG (Sistemas de Información Geográfica), como parte de la necesidad antes mencionada, de representar los diversos aspectos que conforman los sistemas de acueducto y saneamiento que administra la entidad y la relación con el territorio donde están emplazados, creando SIG`s departamentales, los cuales manejan importantes cantidades de datos territoriales de los sistemas y de otros diversos aspectos que le atañen al Instituto.

En el presente documento, se muestran los principales aspectos desarrollados y los resultados del proyecto "construcción y ajuste geográfico de datos espaciales de los sistemas de agua potable y saneamiento del Instituto Costarricense de Acueductos y Alcantarillados", desarrollado en específico en la Dirección de Desarrollo Físico, UEN Programación y Control de la Subgerencia de Ambiente, Investigación y Desarrollo (SAID), con un presupuesto de \$260 mil US dólares y ejecutado mediante licitación abreviada (2013LA-00007-PRI). El proyecto realiza la transformación y mejoramiento de la información cartográfica existente de los sistemas AyA, mediante uso de herramientas SIG.

El proyecto desde su concepción pasa por varias etapas: un diagnóstico de la información espacial de sistemas de acueducto y alcantarillado administrados por AyA, la definición de términos de referencia, la conformación de una metodología para el ajuste y construcción de los datos en un SIG, un proceso de revisión y control de calidad, y por último, la administración, control y distribución de la información generada.

Revista Geográfica de América Central No 55 ISSN 1011-484X, julio-diciembre 2015, pp. 129-152 
Inicialmente en este documento, se presenta la ubicación en el país de los sistemas incluidos en el proyecto, seguido por los objetivos, los aspectos teórico-metodológicos que dan sustento al proyecto, seguidamente por los resultados obtenidos, conclusiones y aplicabilidad de la información generada en los diversos quehaceres institucionales e importancia para los entes externos influenciados por las actividades del Instituto.

\section{Área de Estudio}

El área de estudio incluye sistemas de acueducto y saneamiento administrados por AyA de todas las provincias de Costa Rica (ver mapa 1). La elección de dichos sistemas corresponde a la existencia de información cartográfica digital, es decir, no se incluyen en este proyecto sistemas en donde la información cartográfica disponible es de carácter análogo (en papel) o inexistente.

Mapa 1. Costa Rica: ubicación de sistemas AyA incluidos en el proyecto ajuste geográfico, diciembre 2012.

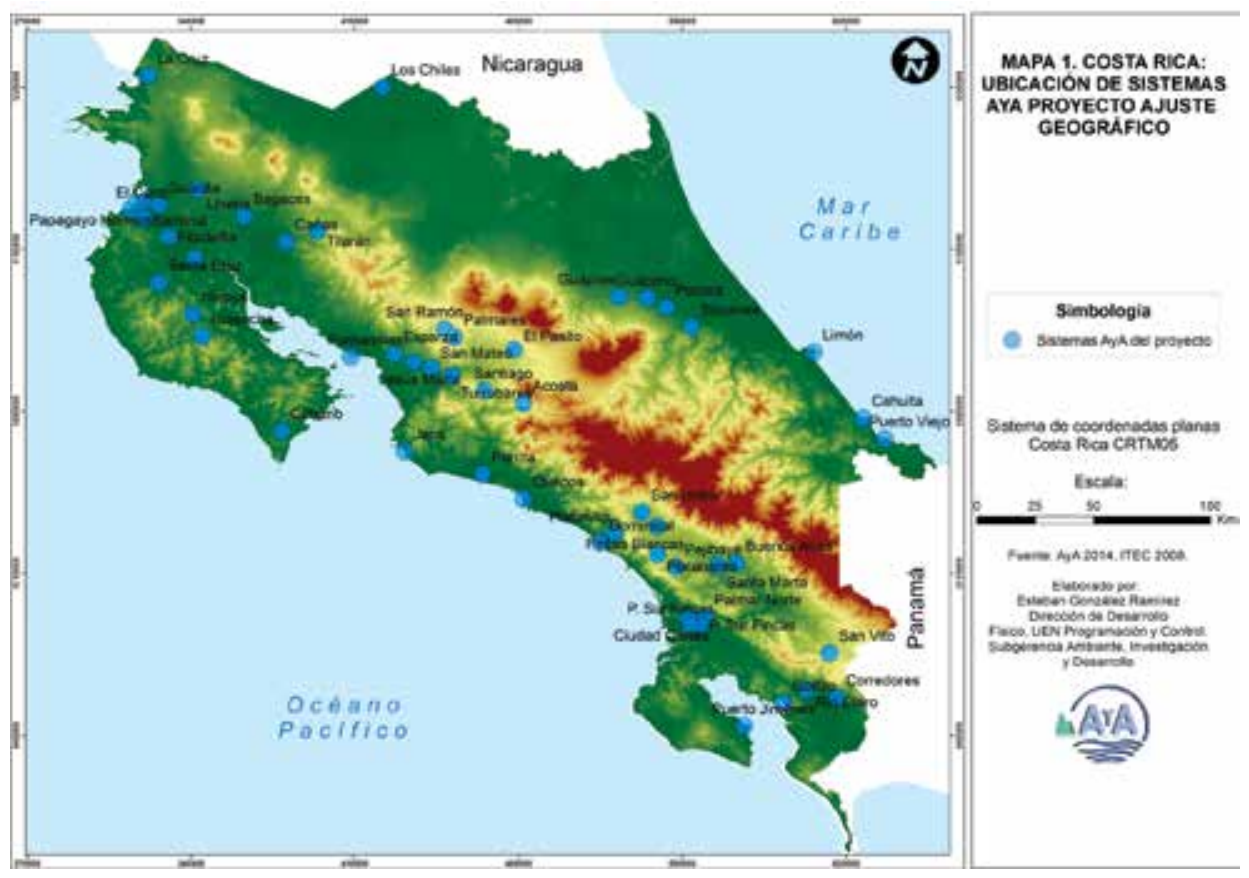

Fuente: AyA: Datos sobre sistemas periféricos de las regiones Brunca, Chorotega, Central Oeste, Pacífico Central, Huetar Atlántica, Subgerencia de Sistemas Periféricos y SAID, 2014. 


\section{Objetivos}

Editar y ajustar a la cartografía del Programa de Regularización y Catastro de Costa Rica (PRCR), la información geoespacial digital existente de los sistemas de agua potable y saneamiento que posee el Instituto Costarricense de Acueductos y Alcantarillados (AyA).

Hacer la conversión de los archivos en versión CAD (dwg, dxf u otro formato) a SHP (shapefile) para su uso en SIG (Sistema de Información Geográfico) de los elementos pertinentes a los sistemas que administra el AyA.

Realizar la homogenización de todos los archivos y productos en el sistema de coordenadas y proyección oficial para Costa Rica CRTM05 y en un sistema universal de coordenadas (coordenadas geográficas WGS 1984).

Elaborar la edición respectiva de los archivos SHP generados de los sistemas AyA y construir una geodatabase respectiva para cada elemento de los acueductos y alcantarillados.

Editar los archivos SHP de los sistemas para que coincidan con la cartografía del PRCR, según lo indican los documentos y planos oficiales del AyA (versiones CAD y otros), o si no existiese, se utiliza la información cartográfica más actual disponible.

\section{Marco Teórico}

La información se ha convertido para la sociedad actual en un bien de alta relevancia, como se observa en diversas corporaciones y entes gubernamentales, que invierten grandes sumas económicas, esfuerzo y dedicación en la gestión de sistemas informáticos.

Para el manejo y análisis de datos ubicados en un territorio, se han desarrollado los llamados Sistemas de Información Geográfica, que desde la óptica de la geografía se conciben como parte de un nuevo campo de conocimiento, denominado nueva geografía aplicada. Así, como una nueva disciplina geográfica, los SIG no pueden considerarse solamente un programa cartográfico, sino que se presentan como herramientas teóricas que permiten pensar y actuar espacialmente (Buzai, 2011).

Como lo explica Moreno (2008), aunque mediante las tecnologías SIG se pueden hacer mapas, esto a la vez, tiene ciertas funciones de dibujo; lo específico y más relevante reside en rasgos como la capacidad de 
almacenar grandes masas de información geo-referenciada o su potencia para el análisis de la misma, que hacen que sea especialmente relevante en abordar problemas de planificación y gestión, en la toma de decisiones.

Según Buzai (2011), la revolución en la forma de pensar que han causado los Sistemas de Información Geográfica, en donde el espacio geográfico es central, se ha basado en cinco aspectos fundamentales de naturaleza espacial: localización, distribución, asociación, interacción y evolución espacial. En este proyecto, varios de estos fundamentos sustentan la base teórica, ya que se usan criterios como localización absoluta y distribución en el territorio para la definición de los productos finales.

Un SIG puede conceptualizarse como un modelo, entendiendo esto como una representación de la realidad, el cual se genera mediante la selección y simplificación de sus partes. El modelo debe tener un sistema de referenciación para que se catalogue como geográfico (Rodríguez, 2008). Para la estandarización del sistema de referencia de los modelos de los acueductos y alcantarillados, se utiliza el sistema de coordenadas local oficial, CRTM05, y además, se realiza una versión paralela en un sistema global, el WGS1984.

El modelo geográfico de la realidad en un SIG, se caracteriza por separar los procesos y objetos existentes en el territorio, descomponiéndolos en partes. Estas partes son el resultado de una disección lógica y consistente de la realidad, que al segregarla se obtienen solamente algunos hechos o aspectos. En conjunto, significa concebir un modelo de la realidad y diseñar una base de datos para contenerlo (Moreno, 2008).

Los sistemas de redes, como los acueductos y alcantarillados, se conforman en un SIG como un modelo de datos que representa una serie de estructuras civiles desagregadas y los diversos accesorios que le componen, emplazados en una posición geográfica específica mediante coordenadas, y dotados de una base de datos o atributos que permite tener grandes cantidades de información relacionada. El concepto de red, en este caso se utiliza para poder comprender más, adecuadamente, y resolver algunos problemas que desde la óptica regional es imposible de resolver. Mediante las redes la apuesta no es la ocupación de áreas, sino la preocupación de activar puntos y líneas o crear nuevos (Durant, Lévy y Retaillé, 1992).

Para la representación de los sistemas de abastecimiento de agua potable y saneamiento, se utiliza un modelo vectorial, ya que las tuberías 
y demás accesorios de los acueductos y los alcantarillados se pueden representar mediante líneas, puntos y áreas. Las redes de agua potable y saneamiento, categorizadas dentro de la teoría geográfica de redes como de Primer Nivel o red real (Somarribas, 2008).

El modelo vectorial se define por usar las figuras de la geometría convencional, puntos, líneas, curvas, polígonos, círculos, elipses o volúmenes para representar las entidades del mundo real (Jones, 1997). El modelo vectorial, como lo indica Rodríguez (2008), resulta particularmente útil para representar entidades geográficas discretas, como el caso de acueductos y alcantarillados.

La información de los sistemas de AyA se almacena en tablas de datos asociadas a los vectores, lo que permite hablar de entidades geográficas y no de meros objetos gráficos, mostrando valores cualitativos y cuantitativos, con la posibilidad de establecer jerarquías y operaciones matemáticas. Los procesos realizados con las tablas de una base de datos están limitados solo por la cantidad de información disponible (Cervera y Rodríguez, 2008).

La información de recursos hídricos y aspectos relacionados con éstos son voluminosos y diversos. Una manera de mejorar la gestión de este tipo de datos ha sido mediante la extensión de Sistemas de Información Geográfica. Como lo indica Solano, Timothy y Morera (1995), la información descriptiva que hay sobre aguas superficiales y subterráneas existente, permite realizar una serie de operaciones que conducen al análisis espacial, el cual debe apoyar acciones como la representación, distribución y medida de datos, la generación de nuevos datos y el análisis espacial de la información.

Los datos de mala calidad afectan, negativamente, a la toma de decisiones en las situaciones cotidianas que requieren información precisa con la que cubrir diversas necesidades de una organización. Las empresas de servicios necesitan mantener sus activos en posiciones precisas, dado que una información incorrecta puede afectar a la finalización de proyectos de construcción o reparación y al envío de notificaciones de corte del suministro a los clientes (ESRI, 2015). Es por ello que la búsqueda de superar los datos de los sistemas AyA resulta esencial para seguir mejorando el servicio en cuanto a continuidad, cantidad y calidad de agua potable y servicio de saneamiento.

134 Revista Geográfica de América Central No 55 ISSN 1011-484X, julio-diciembre 2015, pp. 129-152 
Se utilizan una serie de conceptos de bases de datos, como lo es el término dato, concebido como lo indica Elmasri y Navathe (2007) como los aspectos cuantitativos y cualitativos de una realidad.

En este estudio, se realizaron tareas de entrada manual de datos e importación de archivos de datos digitales; también, se administraron y organizaron los archivos de geodatos de los sistemas AyA. Además, se incluyó trabajo de edición, corrección, integración y geoprocesamiento de los datos, esto abarcó la construcción y modificación de la geometría, las coordenadas, las tablas de datos temáticos, la generación de nuevas unidades espaciales, la normalización de datos dispares, la búsqueda y selección de datos y la obtención de datos derivados (ejecución de cálculos).

\section{Metodología}

Al tratarse de la inclusión de grandes volúmenes de datos observables y que se pueden medir, específicamente, obras civiles que conforman los sistemas de acueducto y saneamiento, la investigación se categoriza como cuantitativa, puesto que es objetiva y orientada a resultados (Barrantes, 2002). Esta definición es importante porque nos indica el abordaje analítico del proyecto, sus estrategias conceptuales, estilo y forma de desarrollo de investigación.

Se usan softwares SIG para el tratamiento y gestión de los datos de los sistemas AyA, tablas u hojas de cálculo y, en general, tecnología informática para la generación de los productos finales, lo cual lo enmarca dentro de este enfoque científico. Este proyecto ha requerido la utilización de software SIG capaz de leer y generar ficheros de información geográfica en diversos formatos (SHP, DXF, TIF, KMZ, etc). Se ha utilizado ArcGIS de ESRI como software básico para la lectura de ficheros, la conversión de formatos y la realización de las tareas de edición y de creación de los productos finales, apoyado por aplicaciones como DWGTrueView y OpenOffice.

Para generar los productos estipulados en los objetivos, en este proyecto se utiliza como base la información cartográfica digital existente en AyA, este se encuentra en formato de software informático para dibujo digital CAD (Computer-aided Design por sus siglas en inglés, diseño asistido por ordenador en español), el cual ha sido el software de dibujo utilizado, regularmente, para crear mapas y planos de sistemas en el 
Instituto. Con esta información, se plantean tres productos específicos: 1 . File Geodatabases (base de datos tipo gdb de ArcGIS para almacenamiento de información) con los elementos de los sistemas AyA desagregados para su uso en SIG; 2. Cartografía CAD a partir de los datos SIG generados en el producto 1; 3. Archivos en formato KML para su uso en Google Earth a partir del producto 1 .

El primer producto, consiste en una serie de File Geodatabases que contienen los archivos Feature Class (shapefiles) correspondientes a los sistemas AyA, generados a partir de los datos CAD mencionados, pero que han sido ajustados a la cartografía más actual disponible, la del Programa de Regularización y Catastro (PRCR), y añadiendo una base de atributos por elemento. Además, se le asigna de un sistema de coordenadas adecuado (CRTM05 y GCS_WGS_1984), parámetros topológicos que controlen los posibles errores de la red y la creación de metadatos para cada archivo. Los archivos shapefile (formato sencillo que se utiliza para almacenar la ubicación geométrica y de atributos de las entidades geográficas), se clasificaron según elemento de red de acueducto y alcantarillado, utilizando el módulo de ArcGIS llamado ArcCatalog. Los archivos shapefile son desplegables y editables en ArcMap de ArcGIS de ESRI (formato dBASE dbf.), a partir de la información original, como se especifica en el siguiente cuadro 1: 
Cuadro 1. Clasificación de las Entidades Geográficas de los Sistemas AyA por archivo de forma

\begin{tabular}{|c|c|}
\hline \multicolumn{2}{|c|}{ Sistemas de acueducto } \\
\hline Entidad Geográfica & Tipo de representación \\
\hline 1. Infraestructura de captación & Punto \\
\hline 2. Tanques & Punto y área \\
\hline 3. Unidades filtrantes & Punto \\
\hline 4. Casetas & Punto \\
\hline 5. Plantas potabilizadoras & Punto y área \\
\hline 6. Estaciones de bombeo & Punto \\
\hline 7. Tuberías & Línea \\
\hline 8. Válvulas & Punto \\
\hline 9. Reducciones & Punto \\
\hline 10. Pasos (elevados u otros) de tuberías. & Línea \\
\hline 11. Tapón & Punto \\
\hline 12. Tee & Punto \\
\hline 13. Unión de tuberías (sobreposición) & Punto \\
\hline \multicolumn{2}{|c|}{ Sistemas de saneamiento (alcantarillado) } \\
\hline Entidad Geográfica & Tipo de representación geográfica \\
\hline 1. Colectores y subcolectores & Línea \\
\hline 2. Plantas de tratamiento & Punto \\
\hline 3. Lagunas de estabilización & Punto y área \\
\hline 4. Estaciones de bombeo & Punto \\
\hline 5. Pasos elevados de colectores y subcolectores & Línea \\
\hline 6. Cajas de registro & Punto \\
\hline
\end{tabular}

Fuente: AyA, Dirección Desarrollo Físico, UEN Programación y Control, SAID, 2014.

El segundo producto, consiste en la creación de una serie de archivos en formato para su uso en software CAD, utilizando como base la versión de GIS del producto 1, para su utilización en actividades de construcción del Instituto.

Por último, un tercer producto es una versión en KML para Google Earth, con la finalidad de mejorar la divulgación de datos de los sistemas a todos los sectores del AyA que no tienen manejo alto en sistemas de información geográficos. 


\section{Fase inicial}

Para este proyecto, inicialmente se realiza un diagnóstico de la información espacial existente, para lo cual se hace un inventario según sistema AyA y se categoriza por su estado, ya sea en digital o en papel, de 170 acueductos y/o alcantarillados administrados por el Instituto.

Los productos de dicho diagnóstico, se resumen en el siguiente cuadro 2. A partir de dicho resultado, se inicia el proceso de constitución de un arquetipo de dato que ayude a homogeneizar la información en un formato adecuado, el cual permite gestionar los datos, mejorar el proceso de actualización y tener adecuada herramienta para la toma de decisiones sobre acueductos y alcantarillados.

Cuadro 2. Estado de la información de los sistemas AyA agosto 2012

\begin{tabular}{|c|c|c|}
\hline Alcantarillado sanitario & 1 & Levantado \\
\hline Planta de tratamiento de aguas residuales & 12 & No existe información \\
\hline Emisario submarino & 1 & No existe información \\
\hline Subtotal & 25 & \\
\hline \multicolumn{2}{|c|}{ Agua Potable } \\
\hline Planta de tratamiento de agua potable & 1 & No existe información \\
\hline \multirow{3}{*}{ Acueductos } & 87 & No existe información \\
\cline { 2 - 3 } & 43 & Digitalizados \\
\cline { 2 - 3 } & 5 & Digital incompleto \\
\cline { 2 - 3 } & 8 & Borrador (papel) \\
\hline Subtotal & 144 & No corresponde a este estudio \\
\hline Gam & 1 & \\
\hline Total & 170 & \\
\hline
\end{tabular}

Fuente: Dirección de Desarrollo Físico, agosto 2012.

En el proceso de diagnóstico, se realizan diversas tareas, como lo es la entrevista a actores clave, recolección de información en tablas, división en categorías de la información y presentación en gráficos y tablas.

\section{Fase de construcción}

Con el diagnóstico elaborado, se procede a incluir los sistemas digitales existentes a un proceso que permita tener información más adecuada y con estándares que ayuden a su interacción en diversos estratos de trabajo e investigación.

Revista Geográfica de América Central No 55 ISSN 1011-484X, julio-diciembre 2015, pp. 129-152 
La totalidad de la información digital existente de los sistemas AyA se encuentra en formato de dibujo informático $\mathrm{CAD}$, elaborado con base en información cartográfica del IGN 1:50000. El proceso de actualización de estos datos, se ha realizado en los últimos años de forma empírica, ya que no se contaba con la información base adecuada, ni con instrumentos de precisión; por lo tanto, el nivel de acierto no es el más preciso. Los datos se encuentran en muchos casos sin un sistema de coordenadas, con simbología heterogénea, repetición de accesorios, falta de elementos y sin reglas que permitan tener una red ideal, para las diversas tareas donde se requiere este tipo de información.

La existencia de información cartográfica actualizada, dentro del marco del Programa Nacional de Regularización y Catastro (PRCR), permite tener una base de mapas de mejor calidad. Es por ello que se decide ajustar, y en casos construir nuevamente, toda la información de los sistemas de AyA a esta nueva base cartográfica, corrigiendo lo posible los errores originados por el uso de información base desactualizada.

Para poder obtener dichos productos, se establece una serie de procedimientos de trabajo que incluyen la edición y automatización de diversas tareas en ArcGIS, implementando, entre otras, las siguientes herramientas y procedimientos:

- Procedimiento de extracción de los elementos de red de acueducto y alcantarillado desde los ficheros CAD originales a formato SHP para proceder a su edición desde ArcGIS.

- Herramientas para la edición gráfica asistida de elementos hasta conformar una red con la topología considerada en el proyecto (unión de tramos de tuberías, colocación de reducciones, tees, válvulas, tapones, tanques, saltos, etc).

- Procedimiento para la incorporación de elementos en la geodatabase con sus características alfanuméricas para la conformación del Producto1.

- $\quad$ Procedimientos para la ejecución del de control de calidad.

- Procedimientos para el cálculo de la orientación y tamaño de los elementos puntuales para la generación de la simbología de accesorios a incorporar en el Producto2.

- $\quad$ Procedimientos para la exportación a CAD, SHP en las proyecciones CTRM05 y WGS84 y a KML para la conformación de los Productos 1,2 y 3 . 


\section{Fase de gestión y administración de los productos}

Terminada la creación de los productos en ArcGIS, se procede a almacenar la información en un dispositivo en red, que permite a usuarios expertos tener acceso para su uso en diversas actividades operativas y de planificación.

El proceso de actualización se lleva a cabo integrando a las regiones en un proceso de capacitación y de acceso a los datos elaborados, teniendo una unidad de coordinación centralizada.

\section{Resultados}

\section{Organización de los datos}

Para cada uno de los elementos de acueducto y saneamiento, se ha constituido una geodatabase en ArcGIS, organizándola según secciones y con tablas específicas y campos de atributos definidos. Los datos organizados, permiten realizar un variado tipo de consultas, cálculos y, la misma es compatible con sistemas SIG de tercera generación para plataformas web. La modernización de la información cartográfica hace que el AyA logre cumplir con una serie de requisitos solicitados a los prestadores de servicios públicos en Costa Rica. En la imagen 2, se observa un ejemplo del modelo visualizado en ArcGIS. 
http://dx.doi.org/10.15359/rgac.2-55.5

Esteban Alberto González-Ramírez. Construcción y ajuste geográfico de datos espaciales de sistemas de agua potable y saneamiento de AyA en Costa Rica

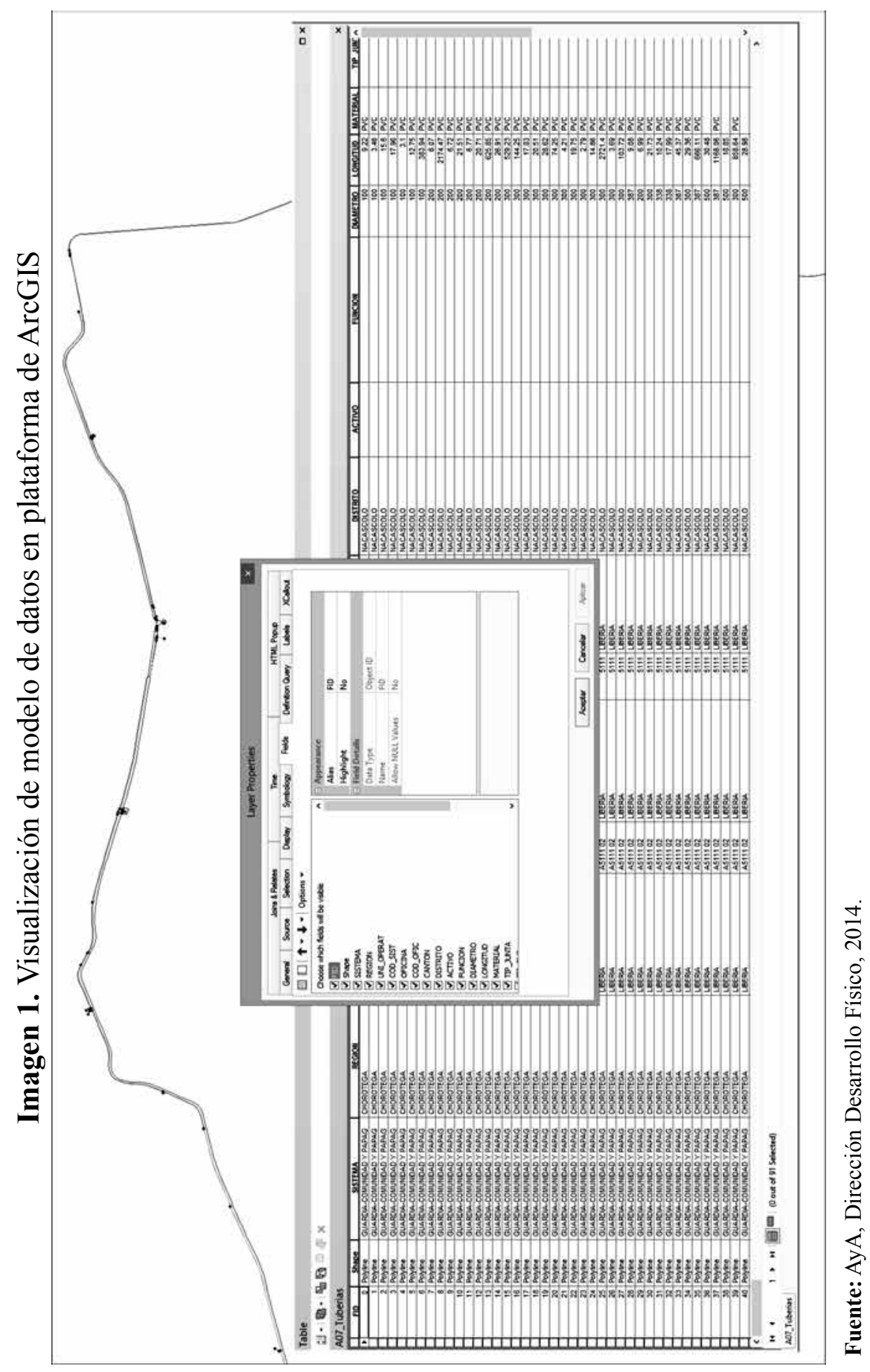

Revista Geográfica de América Central No 55

ISSN 1011-484X, julio-diciembre 2015, pp. 129-152 141

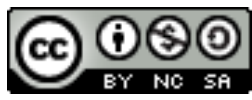




\section{Desarrollo de una herramienta de edición de datos para redes de acueducto y saneamiento.}

Dentro del proyecto, para facilitar se crea una herramienta que permitió editar y visualizar los diversos datos y capas, incluyendo una simbología específica para redes, logrando una mayor productividad en la edición de la información, automatizando la generación de la nuevos datos. En la imagen 2, se presenta la visualización de la herramienta mencionada.

Con la herramienta, es posible visualizar la información de cartografía base y las capas a editar con una simbología adecuada que facilita el trabajo. En el proceso de edición, ajuste y control de calidad, se tienen cargadas en una misma vista las capas con los datos originales de la red y la cartografía e imágenes del Programa de Regularización y Catastro (PRCR).

\section{Proceso de construcción y edición de los datos.}

Con el desarrollo de la herramienta y la interpretación de la información base, se realizan el ajuste y edición para 54 sistemas del AyA, para ello se incluyen las siguientes operaciones para la obtención del producto:

\subsection{Conversión CAD a SHP}

Se realiza la extracción de los elementos de red de acueducto y saneamiento desde los ficheros CAD originales y se transforman a formato SHP para proceder a su edición.

Varios de los ficheros DWG de red originales, se encontraban desplazados respecto a las coordenadas reales de la zona o escalados erróneamente. En estos casos, se realiza un desplazamiento y un cambio de escala en bloque de todo el contenido del DWG para situarlo lo más posible en la zona de trabajo. Después de esto se realiza la conversión a formato shapefile con la herramienta de ArcGIS Extract Data. 
http://dx.doi.org/10.15359/rgac.2-55.5

Esteban Alberto González-Ramírez. Construcción y ajuste geográfico de datos

espaciales de sistemas de agua potable y saneamiento de AyA en Costa Rica

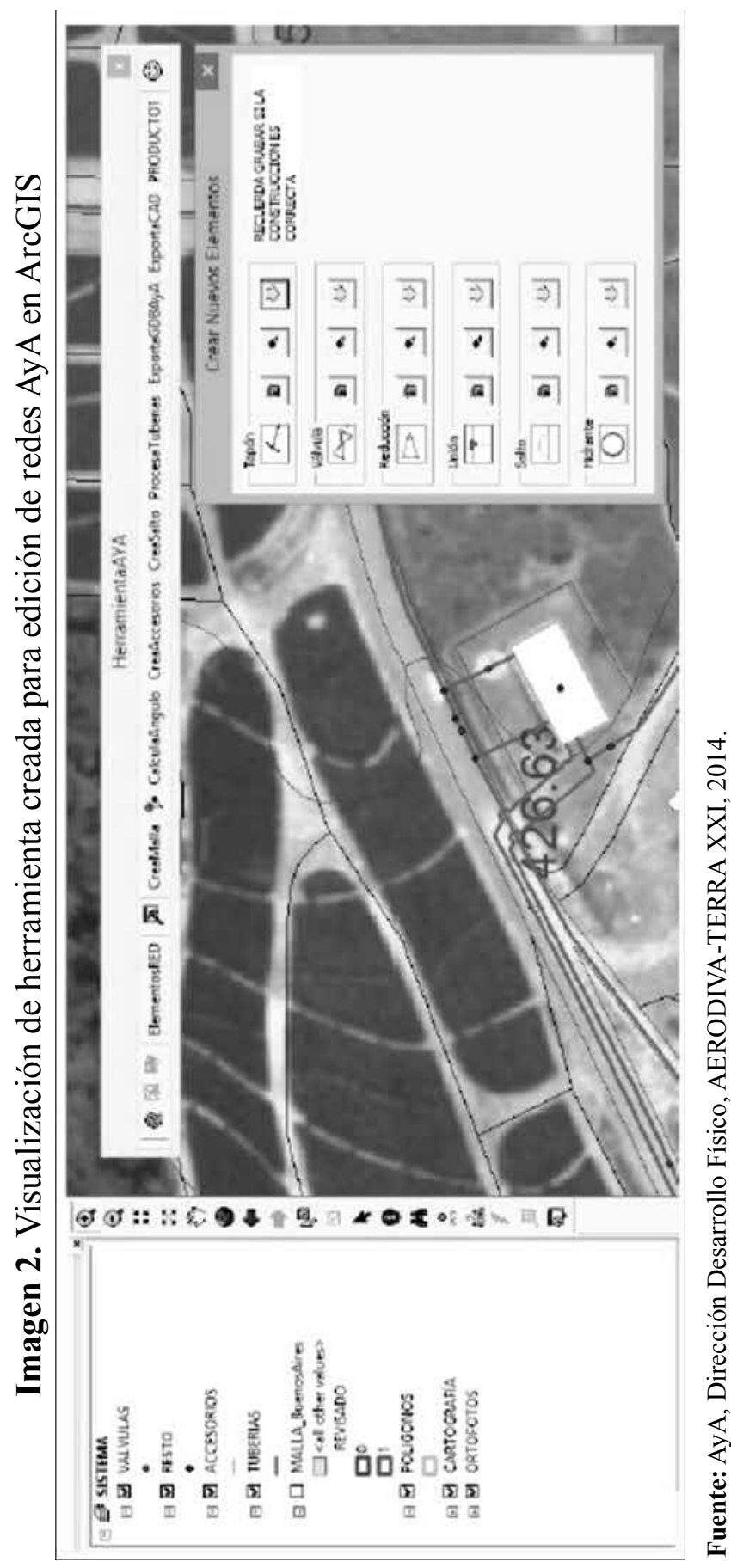




\subsection{Edición de los datos de acueducto y saneamiento}

Se realiza con las herramientas de ArcGIS la respectiva edición de las capas shapefile generadas, con la cual se conforman archivos vectoriales correspondientes a los acueductos y alcantarillados. Se incluyen reglas topológicas contenidas en el software usado para verificar la calidad de los datos. En la imagen 3, se observa un ejemplo de red de un sistema AyA.

3.3. Ajuste a la cartografía más actual disponible: cartas del Programa de Regularización y Catastro (PRCR) de Costa Rica

Para este proceso, se usan las funciones de edición que contiene ArcGIS, asegurando de esta manera, la continuidad de los elementos obtenida en el proceso anterior.

Este proceso de ajuste supone un alto costo de trabajo debido a la interpretación que se debe realizar, respecto a las diferencias existentes entre la representación esquemática utilizada en los $\mathrm{CAD}$ originales y la realidad territorial representada en la cartografía y la ortofoto (ver comparación en la siguiente imagen 4). Se usaron criterios de correspondencia lógica entre lo representado y la realidad detallada en la cartografía del PRCR. 
http://dx.doi.org/10.15359/rgac.2-55.5

Esteban Alberto González-Ramírez. Construcción y ajuste geográfico de datos espaciales de sistemas de agua potable y saneamiento de AyA en Costa Rica

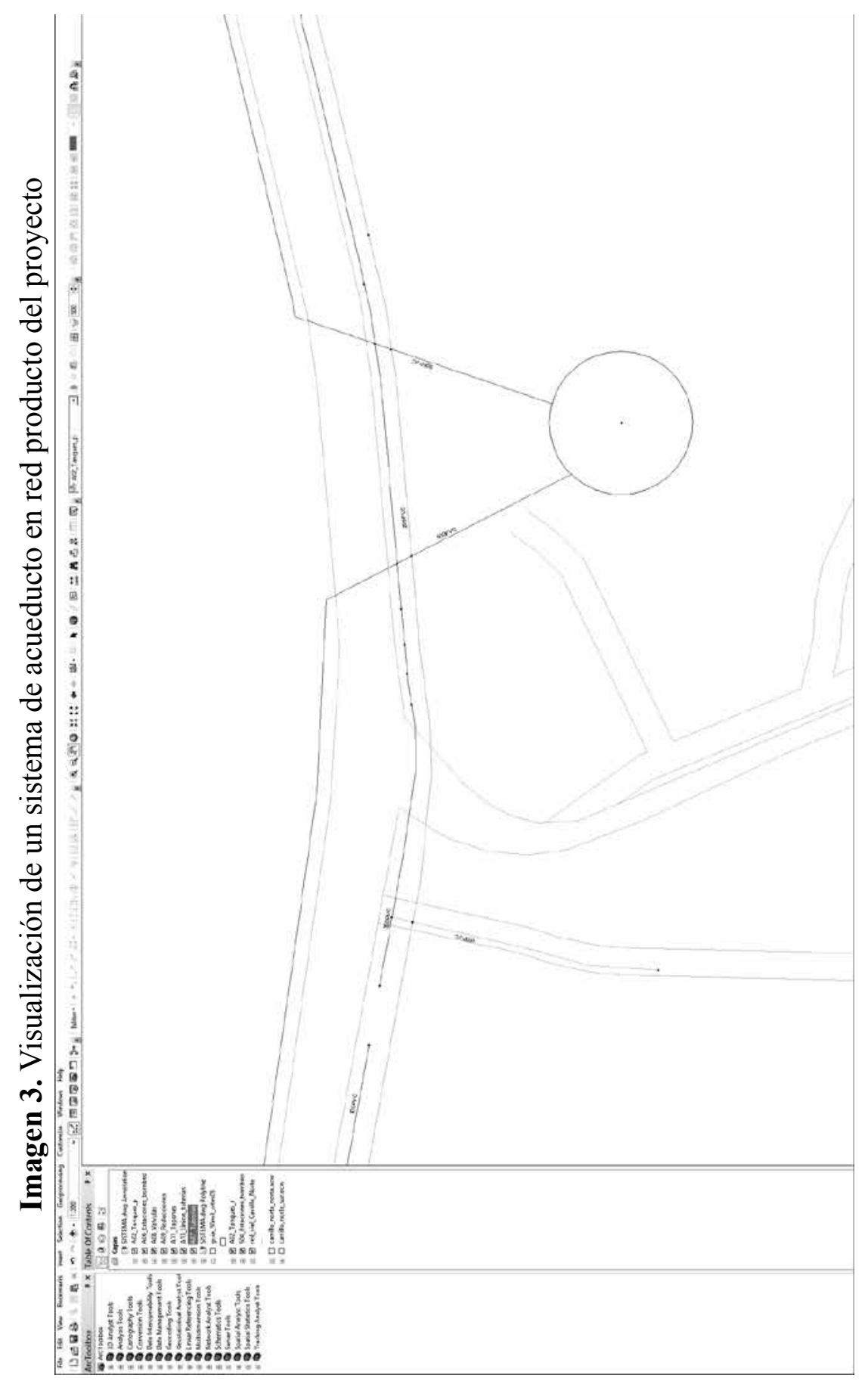

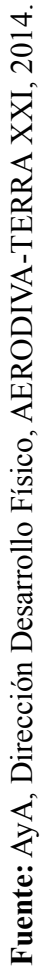


http://dx.doi.org/10.15359/rgac.2-55.5

Esteban Alberto González-Ramírez. Construction and geographic setting of spatial data of drinking water supply and sanitation -AyA, in Costa Rica

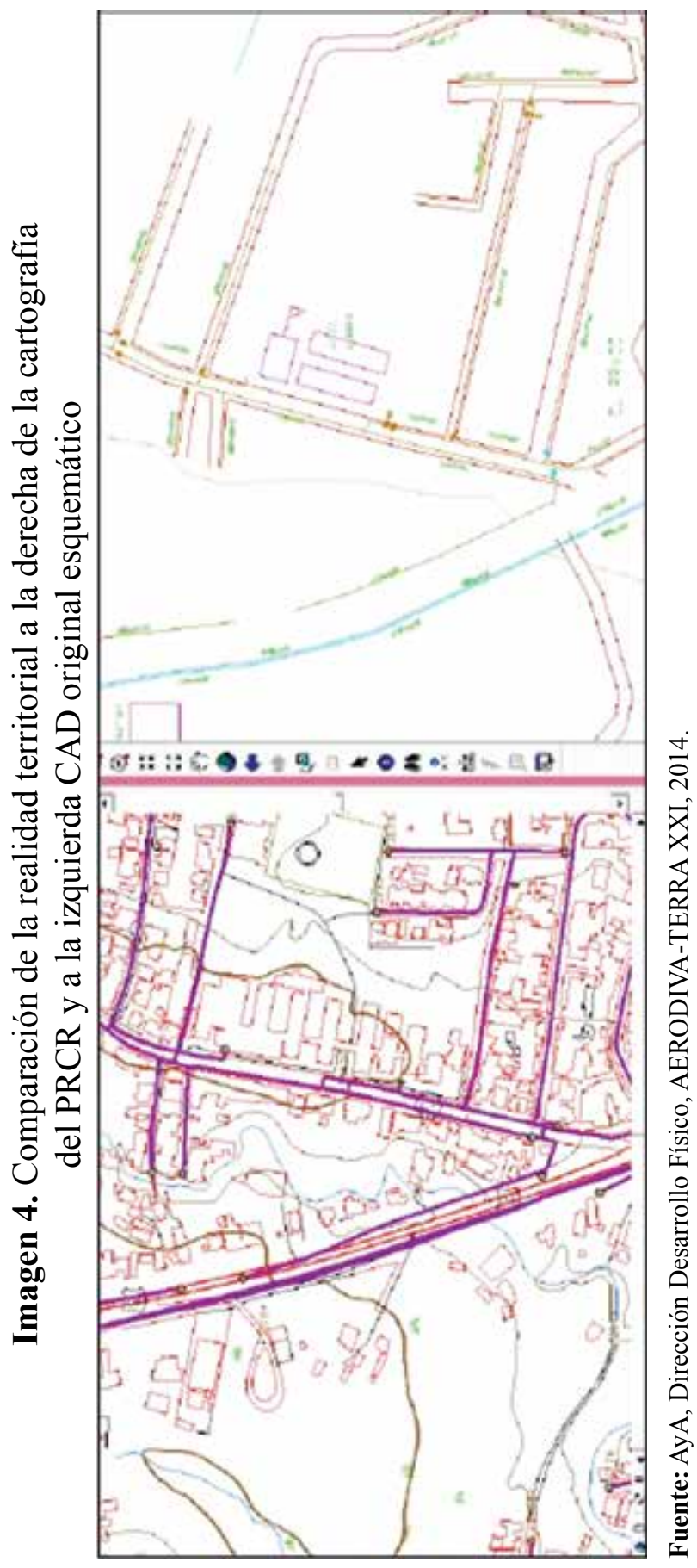

146 Revista Geográfica de América Central No 55 ISSN 1011-484X, julio-diciembre 2015, pp. 129-152 


\subsection{Creación de la geodatabase}

Realizado el proceso de ajuste, se realiza la incorporación de las capas shapefile a una File Geodatabase formato mdb. A esta Geodatabase, se le incorporan una serie de reglas topológicas con la finalidad de evitar los probables errores, como duplicaciones, sobreposiciones, fallas de edición, entre otros, lo que mejora, sustancialmente, la calidad de la información de salida.

Imagen 5. Visualización de la geodatabase en ArcCatalog y topología respectiva

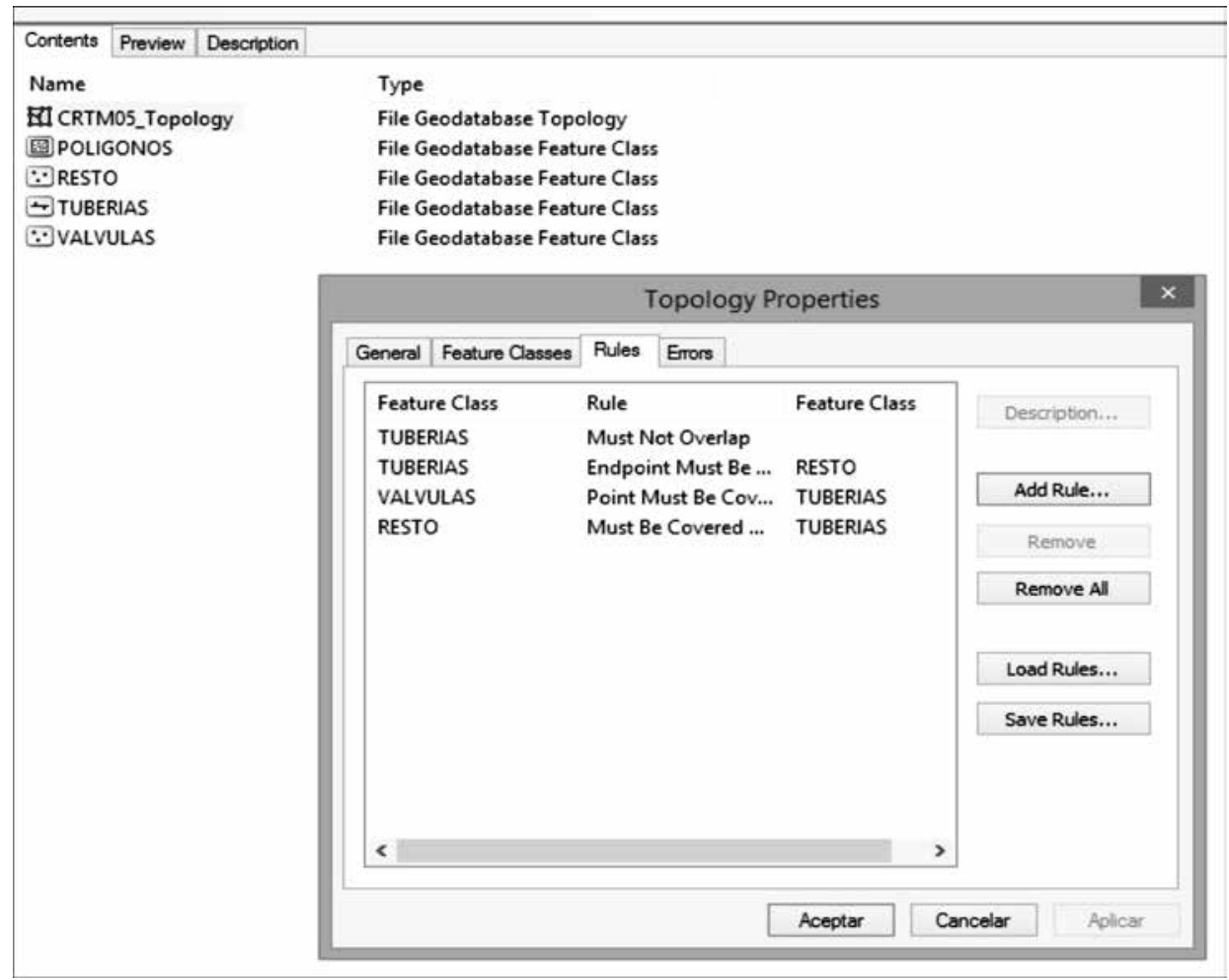

Fuente: AyA, Dirección Desarrollo Físico, AERODIVA-TERRA XXI, 2014.

\section{Productos finales}

A partir de la geodatabase, se realiza la exportación de todos los elementos de la red a los ficheros shapefile (archivos cartográficos), actividad que se realiza mediante la herramienta desarrollada, específicamente, 
para esta actividad y se presentan en versiones de sistemas de coordenadas CRTM05 y WGS84. Un ejemplo se muestra en la siguiente imagen 6.

En el proceso y mediante la función creada, automáticamente, se completan los campos de atributos alfanuméricos de las tablas asociadas a los archivos cartográficos (shapes), tanto las de nomenclatura como nombre del sistema, cantón, distrito, entre otros, como los relacionados con la posición geográfica (latitud y longitud) y las características geométricas, como longitud de tuberías o rotación de válvulas (ver imagen 7).

Imagen 6. Visualización de herramienta para automatización de la creación de productos y ArcCatalog con archivos producto 1

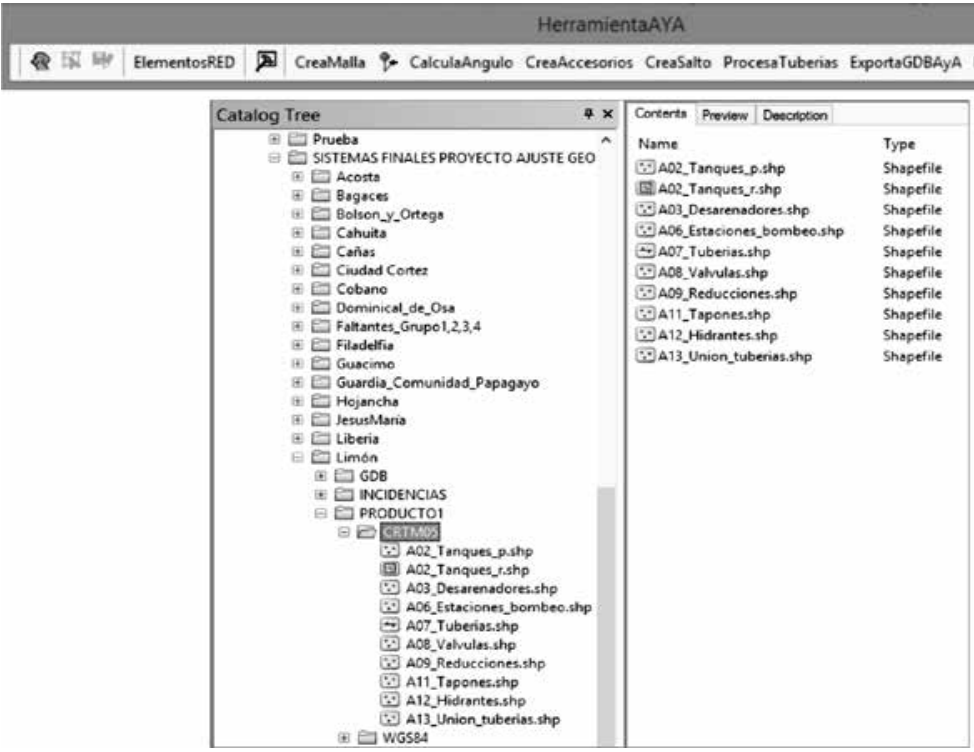

Fuente: AyA, Dirección Desarrollo Físico, AERODIVA-TERRA XXI, 2014.

El segundo producto procede del primero. Es una serie de mosaicos en versión DWG, de los elementos de los sistemas sobre la cartografía catastral del Programa de Regularización y Catastro (PRCR). Todos los elementos de los acueductos y alcantarillados se replican con base en los datos de la geodatabase creada en una nueva versión DWG, formando nuevos ficheros CAD. Para este proceso, se ha desarrollado una herramienta que automatiza el procedimiento, facilitando el trabajo de transformación (ver imagen 7).

148 Revista Geográfica de América Central No 55 ISSN 1011-484X, julio-diciembre 2015, pp. 129-152 
Imagen 7. Visualización de herramienta para automatización de la creación de productos 2 y sistema red en DWG y componentes
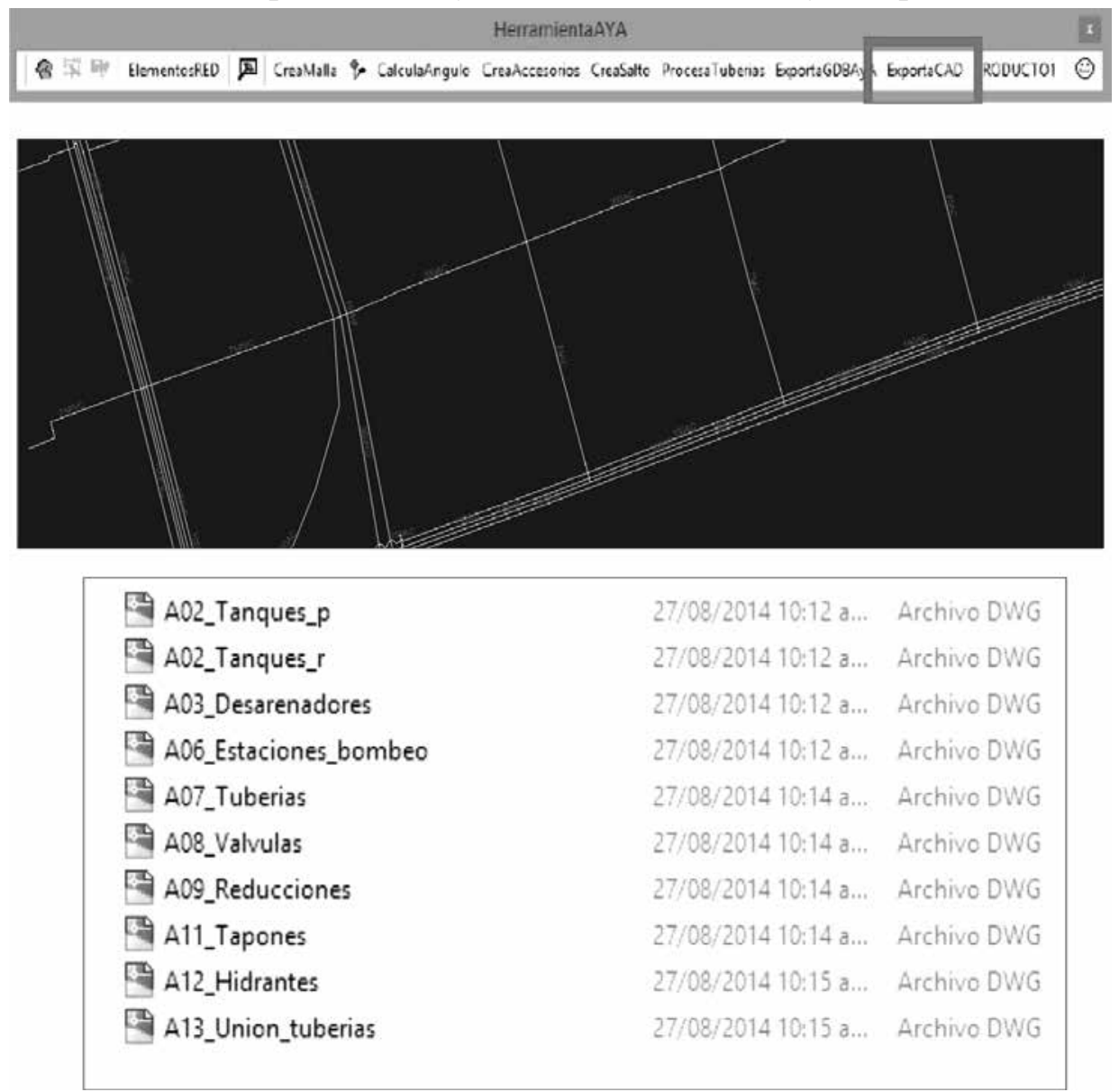

Fuente: AyA, Dirección Desarrollo Físico, AERODIVA-TERRA XXI, 2014.

Un último producto corresponde a una serie de archivos de los sistemas en una versión KML para su utilización en Google Earth. Este producto, se ha concebido con la finalidad de llegar a una serie de usuarios que no tienen acceso a software licenciado o no poseen el manejo de GIS de licenciamiento libre. Un ejemplo de esto se observa en la siguiente imagen 8. 
Esteban Alberto González-Ramírez. Construction and geographic setting of spatial data of drinking water supply and sanitation-AyA, in Costa Rica

Imagen 8. Figura con detalle de los KML de los sistemas AyA

\begin{tabular}{|c|c|c|}
\hline Nombre & Fecha de modefica... & Tipe \\
\hline 2. A01_Infrestructuras_captacion & $24 / 67 / 20141213 \ldots$ & Archimo KS: \\
\hline Q. A02_Tanques.s & $24 / 07 / 20141214 \ldots$ & Archive $\mathrm{KM}$ : \\
\hline Q A02_Tanques, $r$ & $24 / 07 / 20141214 . .$. & Archivo Kat: \\
\hline 2. A06_Estaciones_bombeo & $24 / 07 / 20141214$ & Archivo XM: \\
\hline 9. A07_Tuberias & $74 / 07 / 201412,15 \ldots$ & Archivo KM: \\
\hline 2. A0Q_Volvules & $24 / 07 / 20141215$ & Arehivo XM \\
\hline 9. A09_Reducciones & $2407 / 20141215$. & Archivo 10.4 \\
\hline Q. A11_Tapenes & $24 / 07 / 201412: 15 \ldots$ & Archivo KM: \\
\hline Q. A12.Hidrantes & $24 / 07 / 20141215$ - & Archivo KM: \\
\hline 2. A13_Union_tuberiss & $24 / 07 / 201412: 15 \ldots$ & Archive KM: \\
\hline
\end{tabular}

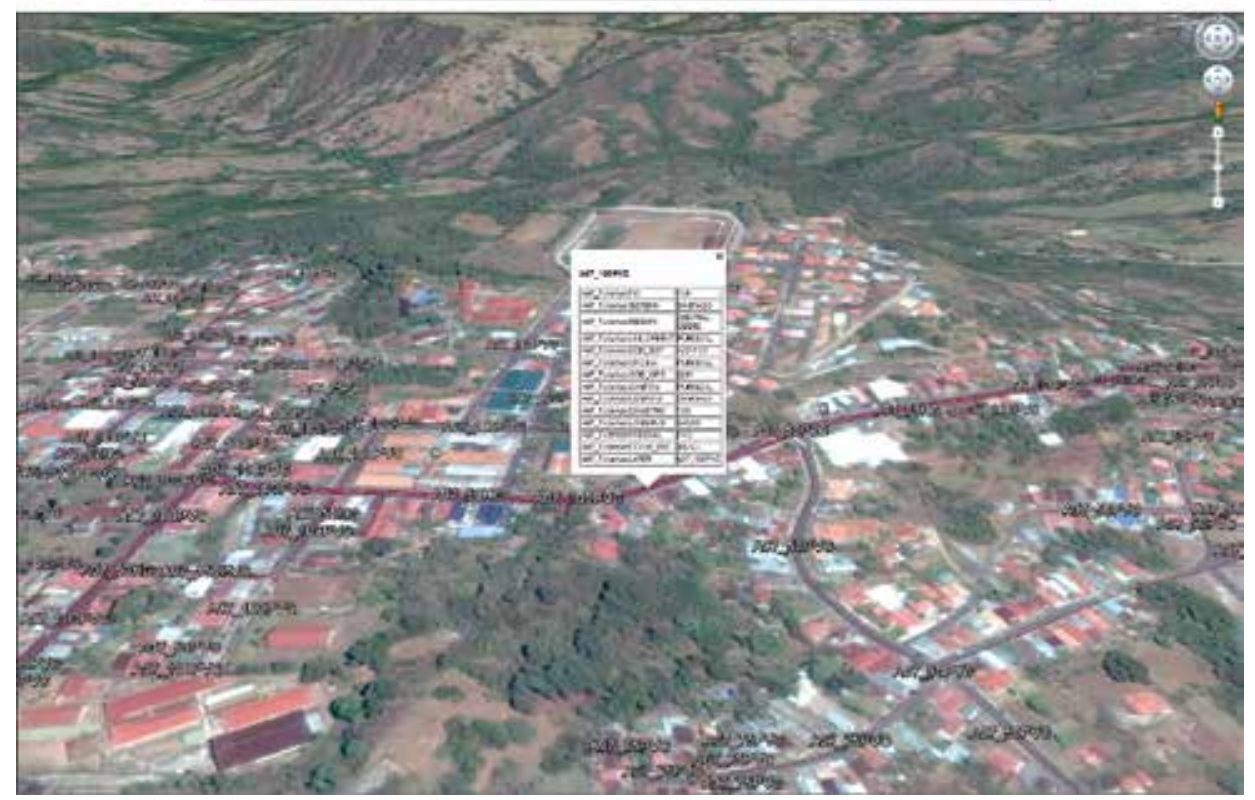

Fuente: AyA, Dirección Desarrollo Físico, AERODIVA-TERRA XXI, 2014.

\section{Discusión de resultados}

El proyecto realizado, permite tener a disposición una versión más adecuada de los datos de obras civiles de los sistemas de acueducto y saneamiento de las principales ciudades de Costa Rica, permitiendo manejar los datos con una base espacial. Para poder resolver problemas relacionados a los sistemas AyA, es necesario contar con datos confiables, por lo que se estima que la información generada en el proyecto ayudará en diversos aspectos a resolver problemáticas relacionadas con tuberías y colectores de saneamiento. 
Los datos generados permiten a mediano plazo tener la base para la constitución de un sistema integrado de información, dentro de los principales usos, es dar una atención más oportuna a los clientes mediante el mejoramiento de la atención domiciliaria, gestión de cortes y re-conexiones, gestionar la deuda, planificar cortes de servicios por zonas, entre otros.

También, permite tener lo necesario para la implementación de operaciones y gerencia de zonas, como el mejoramiento del mantenimiento correctivo de sistemas, las conexiones de nuevas obras, sectorización de redes, entre otros.

En un plazo no muy lejano, los datos generados serán la base para el servicio de mapas que plantea tener la Institución en la web, así como ser la base gestora para el manejo de problemáticas masivas relacionadas con acueductos o saneamiento.

Otro aspecto a futuro, es que con los datos la ejecución de operaciones en redes en el terreno se facilita, teniendo en campo visualización de la red, de cortes, generación de croquis en el terreno, y utilización de tecnología GPS, combinado con los datos para localización, igualmente, con los colectores de saneamiento.

El tipo de dato generado, permite incluir en un corto plazo una serie de vínculos con información documental del instituto, mejorando el nivel de consulta de los usuarios.

\section{Referencias}

AERODIVA- TERRA XXI. (2014). Productos generados en la consultoría para la construcción y ajuste geográfico de datos espaciales de los sistemas de agua potable y saneamiento para el Instituto Costarricense de Acueductos y Alcantarillados. Madrid, España.

Barrantes-Echeverría, R. (2002). Investigación, un camino al conocimiento, un enfoque cualitativo y cuantitativo. San José, C.R.: EUNED.

Buzai, G. D. (2011). Geografía y Sistemas de Información Geográfica (Evolución teórico-metodológica hacia campos emergentes): Conferencia Magistral XIII EGAL Costa Rica 2011. Universidad Nacional de Luján, Programa de Estudios Geográficos, Argentina.

Cervera, B. y Rodríguez, J. (2008). Captura de información alfanumérica. México: Alfaomega. 
Durant, M., Lévy, J., y Retaillé, D. (1992). Le monde, espaces et Systemes, Preses de la Fondation Nacionale des sciencies Politiques et Dalloz. París, Francia.

ESRI (Enviromental Systems Research Institute), ArcGIS Resources. (2015). La importancia del control de calidad. California, EE.UU. Recuperado de http://resources.arcgis.com/es/communities/datareviewer/01rp00000006000000.htm

Instituto Costarricense de Acueductos y Alcantarillados (AyA). (2010). Datos sobre sistemas periféricos de las regiones Brunca, Chorotega, Central Oeste, Pacifico Central, Huetar Atlántica. Subgerencia de Sistemas Periféricos, Pavas, Costa Rica.

Instituto Costarricense de Acueductos y Alcantarillados (AyA) Dirección de Desarrollo Físico. (2014). Datos generados en la consultoría para la construcción y ajuste geográfico de datos espaciales de los sistemas de agua potable y saneamiento para el Instituto Costarricense de Acueductos y Alcantarillados. Pavas, Costa Rica.

Instituto Tecnológico de Costa Rica. (2008). Atlas cantonal digital. Cartago, Costa Rica: Instituto Tecnológico de Costa Rica

Jones, C. (1997). Geographical information systems and computer cartography. Harlow, Longman.

Moreno, A. (2008). Sistemas y análisis de la información geográfica: manual de autoaprendizaje con ArcGIS. México: Alfaomega Grupo Editor.

Rodríguez, J. (2008). El modelo de datos vectorial: características y formatos. México: Alfaomega.

Somarribas, L. (2008). Hacia una geografía de redes: un nuevo paradigma de análisis espacial alternativo al enfoque regional. Revista Geográfica de América Central, 41, 25-52.

Solano, M., Timothy, H. y Morera, C. (1993). Sistemas de Información Geográfica (SIG) como herramienta para el análisis espacial de los recursos hídricos. Universidad Nacional de Costa Rica, Escuela de Ciencias Geográficas, Revista Geográfica de América Central, 27, 123-135.

152 Revista Geográfica de América Central No 55 ISSN 1011-484X, julio-diciembre 2015, pp. 129-152 